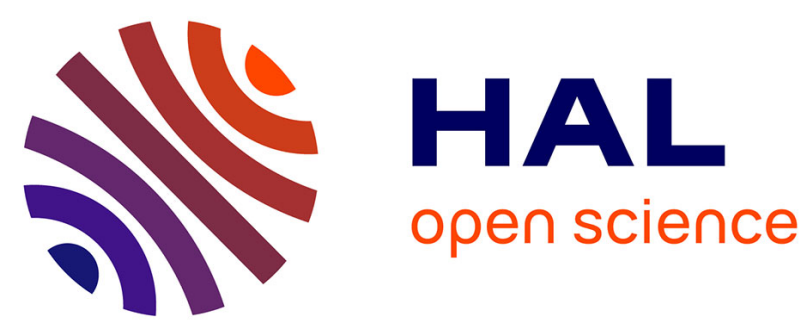

\title{
Synthesis and antioxidant activity evaluation of a syringic hydrazones family.
}

Nadji Belkheiri, Benaissa Bouguerne, Florence Bedos-Belval, Hubert Duran, Corinne Bernis, Robert Salvayre, Anne Nègre-Salvayre, Michel Baltas

\section{- To cite this version:}

Nadji Belkheiri, Benaissa Bouguerne, Florence Bedos-Belval, Hubert Duran, Corinne Bernis, et al.. Synthesis and antioxidant activity evaluation of a syringic hydrazones family.. European Journal of Medicinal Chemistry, 2010, 45 (7), pp.3019-26. 10.1016/j.ejmech.2010.03.031 . inserm-00504802

\section{HAL Id: inserm-00504802 https://www.hal.inserm.fr/inserm-00504802}

Submitted on 22 Jan 2021

HAL is a multi-disciplinary open access archive for the deposit and dissemination of scientific research documents, whether they are published or not. The documents may come from teaching and research institutions in France or abroad, or from public or private research centers.
L'archive ouverte pluridisciplinaire HAL, est destinée au dépôt et à la diffusion de documents scientifiques de niveau recherche, publiés ou non, émanant des établissements d'enseignement et de recherche français ou étrangers, des laboratoires publics ou privés. 
Synthesis and antioxidant activity evaluation of a syringic hydrazones family Nadji Belkheiri, ${ }^{\mathrm{a}, \mathrm{b}}$ Benaissa Bouguerne, ${ }^{\mathrm{a}, \mathrm{b}, \mathrm{c}}$ Florence Bedos-Belval, ${ }^{\mathrm{a}, \mathrm{b}}$ Hubert Duran, ${ }^{\mathrm{a}, \mathrm{b}}$ Corinne Bernis, ${ }^{\mathrm{c}}$ Robert Salvayre, ${ }^{\mathrm{c}}$ Anne Nègre-Salvayre, ${ }^{\mathrm{c}^{*}}$ Michel Baltas,, $\mathrm{b}^{\mathrm{a} *}$

${ }^{a}$ Université de Toulouse ; UPS ; LSPCMIB (Laboratoire de Synthèse et Physico-Chimie de Molécules d'Intérêt Biologique) ; 118, Route de Narbonne ; F-31062 Toulouse Cedex 9, France.

${ }^{b}$ CNRS ; LSPCMIB (Laboratoire de Synthèse et Physico-Chimie de Molécules d'Intérêt Biologique) ; 118, Route de Narbonne ; F-31062 Toulouse Cedex 9, France.

${ }^{c}$ INSERM I2MR 858, IFR 31 CHU Rangueil, L3 building, Avenue Jean Poulhès BP 84225 31432 Toulouse Cedex 4, France

Corresponding author: M. Baltas: corresponding author; Tel. : 0033 (0)5 615562 89; Fax: 0033 (0)5 615560 11; E-mail : baltas@chimie.ups-tlse.fr 


\begin{abstract}
A novel series of hydrazones derived from syringaldehyde and their antioxidant properties have been explored. Several employed methods such as scavenging effect on 2,2diphenyl-1-picrylhydrazyl (DPPH) radical and 2,2,'-azinobis(3-ethylbenzthiazoline-6-sulfonic acid $\left(\mathrm{ABTS}^{\bullet+}\right)$ radical cation expressed as Trolox equivalent antioxidant capacity (TEAC), inhibition of superoxide anion $\left(\mathrm{O}_{2}{ }^{-*}\right)$ generation and of human cell-mediated low-density lipoprotein oxidation (monitored by the formation of TBARS) exhibited their potent antioxidant properties. The carbonyl scavenger efficacy was also evaluated by measuring the ability to decrease the protein carbonyl content in cells challenged with oxidized LDL. In this report, we discuss about the synthesis of hydrazones and their dual biological role, antioxidant and carbonyl scavenger for further application in atherosclerosis.
\end{abstract}

Keywords: Syringic hydrazones, antioxidants, oxidative stress, carbonyl stress, LDL oxidation, atherosclerosis. 


\section{Introduction}

Oxidative stress is a well-known mechanism that is responsible for the development of vascular damage. Different pathogenic stimuli involved in cardiovascular diseases, such as activated macrophages, hyperglycaemia, oxidized low density lipoprotein (LDL), exert their harmful effects, at least partially, through an increased local generation of reactive oxygen species. Reactive Oxygen Species (ROS) are normally produced throughout oxygen metabolism and play a major role in physiological and pathological cell redox signalling.[1] Oxidative stress results from an imbalance between $\mathrm{i} /$ an overproduction of ROS by the different cellular sources such as the mitochondrial respiratory chain, nicotinamide adenine dinucleotide phosphate hydride oxidases (NADPHOXs or NOXs), xanthine oxidase, lipoxygenases, cytochromes $\mathrm{P} 450$, and other oxidases and ii/ decreased cellular and plasma antioxidant defenses [1]. Besides the direct free radical attack of cellular components, oxidative stress induces a lipid peroxidation of cellular membranes resulting in the generation of reactive carbonyl compounds (RCC) that react rapidly with free amino groups and thiol residues on proteins thereby forming adducts involved in the "carbonyl stress" [2,3]. Both oxidative and carbonyl stress alter protein function and trigger the accumulation of modified proteins, which results in inflammation and apoptosis $[3,4]$. ROS and RCC are implicated in the pathophysiology of ageing and oxidative stress-associated pathologies such as diabetes, neurodegenerative diseases, atherosclerosis and cardiovascular complications [5-6]. Low density lipoproteins (LDL) become atherogenic after undergoing oxidation within the vascular wall, in the presence of transition metals and ROS generated by vascular cells [7]. Oxidized LDL are involved in the formation of early atherosclerotic lesions (foam cell deposition as fatty streaks), and in their evolution toward more advanced states characterized by endothelial dysfunction, chronic inflammatory responses and toxic events leading to plaque rupture and thrombosis [7-8]. 
Antioxidants inhibit the generation of ROS and the subsequent formation of lipid peroxidation products, thereby preventing both oxidative and carbonyl stress. Most antioxidants prevent LDL oxidation in cell-dependent and cell-free systems, and delay the formation of atherosclerotic lesions in animal models for atherosclerosis such as apoE-/- mice.[9] Discrepancies exist about their efficiency against atherosclerosis in humans; this resulting at least in part, from their bioavailability within the plaque, their chemical nature and their inability to scavenge RCCs, once adducts are formed on proteins [10]. However antioxidants remain very efficient in preventing the early atherosclerotic lesions and inflammatory events implicated in the evolution of the lesions toward more advanced states [9-10].

We have recently reported that hydrazine derivatives such as hydralazine, exhibit efficient antiatherogenic activity mediated by their carbonyl scavenger activity [11]. However their antioxidant capacity is moderate, limiting their use as antiatherogenic agents. Moreover, we have also reported that phosphonocinnamic derivatives obtained by reaction between syringaldehyde and activated phosphonates possess interesting dual biological activities as antioxidant and cytoprotective properties [12]. More recently, we have designed biaryl compounds based on 5,5'-bisvanillin scaffold possessing identical or different substitutions: the known phosphonodiester and/or fluorophosphonoester moiety and a hydrazone function [13]. The best compounds in term of antioxidant and cytoprotective effects was found to be the symmetrical compound bearing two hydralazine scaffolds while all other ones were much less potent. In continuation of this work and in order to get insight on the efficiency of the hydrazone functionality, we report here the synthesis of simpler monomeric hydrazonoderivatives obtained through syringaldehyde coupling with a series of hydrazines and their ability to block oxidation parameters. Their potency have been evaluated through their impact on the 2,2-diphenyl-1-picrylhydrazyl (DPPH) assay [14], Trolox equivalent antioxidant capacity (TEAC) [15], the carbonyl and $\mathrm{O}_{2}{ }^{\bullet-}$ scavenging activities. 


\section{Chemistry}

Syringic hydrazones described were readily synthesized according to the procedures outlined in Scheme 1. Commercially available syringaldehyde was refluxed in absolute ethanol with the different hydrazines during 6 to 12 hours. The reaction was followed by TLC until completion. The solution was then cooled to room temperature and the resulting precipitate was collected by filtration to provide the corresponding hydrazones as hydrochloride salt or not, depending on the nature of commercially available hydrazines in good to excellent yield. (Scheme 1)

\section{Pharmacology}

\subsection{Scavenging radical activity on 2,2-diphenyl-1-picrylhydrazyl (DPPH) radical}

Radical scavenging potency of the compounds tested was assessed in vitro by the DPPH assay. DPPH is one of the few stable and commercially available organic nitrogen radicals. DPPH radicals are considered as a representative method for the preliminary screening of compounds able to scavenge activated oxygen species, since they are more stable and easier to handle than oxygen free radicals and DPPH assay has been largely used to measure the antioxidant activity of different phenolic compounds.

This method is based on measuring the continual absorbance decrease of the ethanol solution of the stable free nitrogen radical DPPH at $517 \mathrm{~nm}$, in the presence of the derivatives tested. All tested hydrazones present UV absorbance between 230 and $350 \mathrm{~nm}$, and do not interfere with the kinetic measurement of DPPH absorbance decrease at $517 \mathrm{~nm}$. The radical scavenging activity was expressed as the molar amount of antioxidant necessary to decrease the initial DPPH concentration by 50\% (efficient concentration) EC50. (Figure 1) 
$\mathrm{The} \mathrm{DPPH}^{\bullet}$ absorbance decrease in presence of antioxidants is due to hydrogen transfer from the antioxidant, thus forming the DPPH-H stable compound. Ethanolic DPPH ${ }^{\bullet}$ solutions used at different concentrations were stable (checked over a $60 \mathrm{~min}$ period). A significant decrease in the concentration of $\mathrm{DPPH}^{\cdot}$ radical due to the scavenging ability of some of the antioxidants was observed. The $\mathrm{DPPH}^{\circ}$ absorbance values were respectively $0.16,0.34,0.38$, $0.38,0.48,0.50,0.64,0.65,0.75,0.92$ and 1.00 with $\mathrm{DPPH}^{\bullet}$ initial optical absorbance of 1.01 at $517 \mathrm{~nm}$. The IC50 values were determined for all compounds and reported in Figure 1. Compounds possessing a pyridine ring adjacent to the carbonyl group have the lowest IC50 values among the synthesized hydrazones $(17$ and $18 \mu \mathrm{M}$ for compounds $\mathbf{8}$ and $\mathbf{7}$ respectively).

As shown in scheme 2 for compound $\mathbf{8}$, the hydrogen atom of the isoniazid moiety in $\alpha$ position from the hydrazone function is highly activated. Compound $\mathbf{8}$ may easily undergo abstraction of one hydrogen atom from this position. Hydrogen atom abstraction from phenolic ring under the examined conditions is quite difficult due to its strong intramolecular bond with the adjacent methoxy groups. As a consequence the reaction of DPPH radicals may create nitrogen radical on the antioxidant considered. This is in agreement with the recent work of Dolenc and al.[17] concerning the autoxidation process of hydrazones. After studying structurally different hydrazones, the authors proposed for those possessing at least one hydrogen atom on nitrogen, the formation of $\mathrm{N}^{\bullet}$ centered radical (hydrazonyl radical) which is stabilized through conjugation forming delocalisated $\mathrm{C}^{\bullet}$ centered radicals. The different $\mathrm{DPPH}^{\bullet}$ scavenging properties (IC50) of the synthesised hydrazones may reflect the relative ability for the N-H hydrogen atom abstraction from the considered compound. 


\subsection{Scavenging radical activity on 2,2'-azinobis(3-ethylbenzthiazoline-6-sulfonic acid)}

$\left(\right.$ ABTS $\left.^{+}\right)$radical cation

The antioxidative activity of syringic hydrazones was also evaluated by measuring the Trolox equivalent antioxidant capacity values (TEAC).This method measures the relative ability of antioxidant substances to scavenge the radical cation 2,2'-azinobis(3-ethylbenzthiazoline-6sulfonic acid) $\left(\mathrm{ABTS}^{\bullet+}\right)$ compared with a standard amount of the synthetic antioxidant Trolox (6-hydroxy-2.5,7,8-tetramethylchroman-2-carboxylic acid), a vitamin E analogue. The TEAC value is defined as the concentration of standard Trolox with the same antioxidant capacity as $1 \mathrm{mM}$ concentration of the antioxidant compound under investigation. This assay is based on the generation and detection of a colored long-lived specific radical cation; ABTS is chemically oxidized by potassium persulphate to give the relatively stable (over 24 hours) ABTS radical. The concentration of $\mathrm{ABTS}^{\circ+}$ is measured at $734 \mathrm{~nm}$.[16] Thus the antioxidantinduced reduction of the $\mathrm{ABTS}^{{ }^{+}}$concentration is directly related to the antioxidant capacity of the compound being tested.

ABTS radicals are involved in an electron transfer process. All screening compounds exhibited at various levels radical cation scavenging activity. There were no differences between the TEAC values for the syringic hydrazones at $1 \mathrm{~min}, 4 \mathrm{~min}$ and $6 \mathrm{~min}$ (data not shown).

As seen in figure 2, compounds 7 and $\mathbf{8}$ were the most effective ABTS $^{{ }^{++}}$radical scavengers depending on concentration range $(0-15 \mu \mathrm{M})$. ABTS $^{{ }^{+}}$absorbance decreases significantly with all hydrazone concentrations, the effect being less pronounced for hydrazone $\mathbf{8}$ at concentrations above $12 \mu \mathrm{M}$. The scavenging effects on $\mathrm{ABTS}^{\cdot+}$ decreased in the order $\mathbf{7}>\mathbf{8}$ $>\mathbf{9}>\mathbf{1 0}>\mathbf{4}=$ Trolox $=\mathbf{5}>\mathbf{2}>\mathbf{3}>\mathbf{1}>\mathbf{6}$. 
Table 1 summarised the results obtained in terms of Trolox equivalents capacity. Three hydrazones, among the tested ones, $7, \mathbf{8}$ and $\mathbf{9}$ possess TEAC values more than $15 \%$ of the Trolox value while the other compounds possess equivalent or inferior values. This might be due to a better transfer of radical and better resonance stabilisation of the radical species for theses compounds. (Table 1)

\subsection{Effect of syringic hydrazones on LDL oxidation and superoxide anion generation}

The protective effect of syringic hydrazones was studied on LDL oxidation induced either by cell contact with human microvascular endothelial cells (HMEC-1) (this system allows to mimic the pathophysiological events occurring in the vascular wall) [18], or by two "cell-free systems" e.g. i/ oxidation by copper ions at $37^{\circ} \mathrm{C}$, which initiates radical chain reaction with lipoperoxides preformed in LDL, thereby generating peroxy radicals, hydroxides, hydroperoxides and aldehydes [19], and ii/ UV-C irradiation at $254 \mathrm{~nm}$, which generates polyunsaturated fatty acid (linoleate) hydroperoxides, as well as oxysterols and aldehydes [20]. Preliminary self-toxicity experiments indicated that some syringic hydrazone monomers exhibit a slight cytotoxicity for HMEC-1, at concentrations higher than $100 \mu \mathrm{M}$. As a consequence, the protective effect of the different agents on LDL oxidation was studied and compared to that of Trolox [21] at a fixed concentration of $10 \mu \mathrm{M}$. (Table 2)

The data presented in Table 2, indicate that all the monomers except hydrazones $\mathbf{7}$ and $\mathbf{8}$ exhibited a comparable inhibitory effect on cell-induced and UV-induced LDL oxidation, which was similar to that of Trolox. All the agents blocked effectively copper-induced LDL oxidation, except compounds $\mathbf{7}$ and $\mathbf{8}$ which were inefficient (note that isoniazid and syringaldehyde alone did not block copper- nor UV-induced LDL oxidation, data not shown). All the evaluated compounds blocked efficiently cells-induced LDL oxidation at the same level observed for 5,5'-bisvanillin bearing two hydralazine scaffolds [13]. Syringic 
hydrazones inhibited by $30-50 \%$ the generation of $\mathrm{O}_{2}{ }^{--}$by HMEC-1, in agreement with the implication of $\mathrm{O}_{2}{ }^{--}$in LDL oxidation, as reported.[18] However, the inhibitory effect of syringic hydrazone on $\mathrm{O}_{2}{ }^{\circ-}$ generation was lower than that observed with Trolox (Table 2), suggesting that other ROS are implicated in cell-mediated LDL oxidation.

Since oxidized LDL are highly cytotoxic for vascular cells, we measured the cell viability of HMEC-1 at the end of the cell-induced LDL oxidation process by 3-(4,5dimethylthiazol-2-yl)-2,5-diphenyltetrazolium bromide (MTT) assay. As shown in Table 2, cell-oxidized LDL exhibited a strong cytotoxicity for HMEC-1, which was protected by all the newly synthesized syringic hydrazones, and was similar to the protective effect of Trolox but less to the one of 5,5'-bisvanillin bearing two hydralazine scaffolds (55-79\% vs 82\%) [13].

We then compared the antioxidant ability and cytoprotective effect to the constitutive frame, (Figure 3) of three agents possessing different hydrazone links: compound $\mathbf{1}$ with hydralazine frame (only hydrazone link), compound $\mathbf{6}$ with aminoguanidine moiety (free $\mathrm{NH}$ ) and compound $\mathbf{8}$ with isoniazid fragment (hydrazide link). Likewise, syringaldehyde, aminoguanidine or isoniazid did not block cell-mediated LDL oxidation, while syringic isoniazid hydrazone $\mathbf{8}$ and syringic aminoguanidine hydrazone $\mathbf{6}$ were very efficient (figure 3A). The ability of these agents to inhibit LDL oxidation and $\mathrm{O}_{2}{ }^{\circ}$ generation mediated by HMEC-1 was tested, and compared to the results obtained in the presence of native hydrazines. Interestingly, neither syringaldehyde, nor aminoguanidine and isoniazid were protective against oxidized LDL-induced cytotoxicity, while their respective monomeric derivatives $\mathbf{6}$ and $\mathbf{8}$ were strongly efficient for inhibiting LDL oxidation and cytotoxicity as well (figure 3). It is to note that hydralazine and its related monomer exhibited comparable inhibitory properties against LDL oxidation and cytotoxicity (figure 3B), in agreement with 
previous results showing the strong antioxidant and cytoprotective properties of hydralazine [11].

\subsection{Carbonyl scavenger properties}

Carbonyl stress is characterized by the formation and the accumulation of adducts formed on proteins by aldehydes generated during the oxidation of polyunsaturated fatty acids.[3,5] The formation of adducts on proteins lead to a progressive protein dysfunction finally resulting in apoptosis [11]. As an exemple, 4-hydroxynonenal (4-HNE) is a major aldehyde generated during the peroxidation process of polyunsaturated fatty acid in LDL [11]. 4-HNE is involved in the carbonyl stress, as it forms adducts on proteins and induces cytotoxicity. The protective effect of syringic hydrazones against carbonyl stress, was evaluated on their ability to block the cytotoxicity induced by 4-HNE $(25 \mu \mathrm{M})$ in HMEC-1. As shown on Figure 4, most agents almost completely prevented 4-HNE-induced cell death, and were much more efficient than hydrazine derivatives used alone, particularly isoniazid. Syringic acid (used for the synthesis of syringic hydrazones) and (used as antioxidant) were unefficient in preventing 4-HNEinduced cytotoxicity.

\section{Conclusion}

In this work, we have evaluated the antioxidant and carbonyl scavenger capacities of a series of newly synthesized syringic hydrazones. They have been obtained easily through coupling reaction between syringaldehyde and suitably chosen hydrazine possessing electron withdrawing or electron attracting groups. Their potency against the oxidative stress was adressed by different means: via the 1,1-diphenyl-2-picrylhydrazyl (DPPH), Trolox equivalent antioxidant capacity (TEAC), inhibition of LDL oxidation and of superoxide anion 
generation. These new compounds are of interest because all of them were found to be effective antioxidants possessing reducing power on the $\mathrm{DPPH}^{\circ}$ and $\mathrm{TBARS}^{\circ}$ assays, depending on their capacity of an N-H hydrogen abstraction and on resonance stabilisation of the phenoxy radical. In the TBARS assay the compounds seemed at most equivalent to the Trolox scavenging capacity, which does not reflect their overall antioxidant activity possibly due to other mechanisms involved. Moreover, these agents exhibit a strong carbonyl scavenger activity evidenced by their ability to block 4-HNE-mediated cell death (which is not prevented by antioxidants, as reported here with Trolox).

Altogether the data suggest that the inhibition of cell-mediated LDL oxidation by syringic hydrazones is higher than that observed with the initial hydrazine compounds, and probably does not result from a simple ROS-scavenger or antioxidant activity in the culture medium, but rather from the inhibition of cell-mediated ROS generation, in agreement with the inhibition of cellular NADPH oxidase by hydralazine previously reported [23]. It is hypothesized that the protective (antiatherogenic) effect of syringic hydrazones should result from their carbonyl scavenger properties and the potent antioxidant effect against LDL oxidation (and $\mathrm{O}_{2}{ }^{\circ-}$ generation), resulting from the combination of syringaldehyde with hydrazine compounds (isoniazid) or aminoguanidine, while these agents alone exhibit poor antioxidant activity. It is to note that Trolox, though strongly efficient antioxidant, is unable to trap and neutralize carbonyl compounds and aldehydes issued from lipid peroxidation, once formed, in contrast to syringic hydrazones which share both antioxidant and carbonyl scavenger properties. These new and original hydrazone drugs could constitute a new class of agents sharing both valuable antioxidant and carbonyl scavenger activities, with potential application in oxidative stress-associated pathologies, and particularly atherosclerosis.

\section{Experimental section}




\subsection{Chemistry}

A mixture of hydrazine (1 equivalent) and syringaldehyde (1 equivalent) was dissolved in absolute EtOH $(40 \mathrm{~mL} / \mathrm{mmol})$. The solution was heated under reflux for 6 to 24 hours depending on hydrazine used and then cooled to $25{ }^{\circ} \mathrm{C}$. The precipitated solid was collected by filtration and rinsed thoroughly with $\mathrm{EtOH}$ to afford the corresponding hydrazone. If no precipitate was observed, the reaction mixture was concentrated under reduced pressure and the crude residue was purified by silica gel chromatography affording the corresponding hydrazone.

(E)-4-Hydroxy-3,5-dimethoxybenzaldehyde phthalazin-1-ylhydrazone hydrochloride (1) Yellow solid; yield 75\%; m.p. $154-156{ }^{\circ} \mathrm{C}$; IR (KBr) $\vee \mathrm{cm}^{-1}: 3415(\mathrm{O}-\mathrm{H}), 1630(\mathrm{C}=\mathrm{N})$, $1619(\mathrm{C}=\mathrm{C}), 1592(\mathrm{C}=\mathrm{C}$ arom. $), 1516(\mathrm{C}=\mathrm{C}$ arom. $), 1464(\mathrm{C}=\mathrm{C}$ arom. $), 1222(\mathrm{C}-\mathrm{O}), 1122$ (C-O) ; ${ }^{1} \mathrm{H} \mathrm{NMR}\left(\mathrm{CDCl}_{3}, 300 \mathrm{MHz}\right) \delta \mathrm{ppm}: 3.84\left(\mathrm{~s}, 6 \mathrm{H}, \mathrm{OCH}_{3}\right), 7.38\left(\mathrm{~s}, 2 \mathrm{H}, \mathrm{H}_{2,6}\right), 8.15(\mathrm{td}$, $\left.\mathrm{J}=8.2 \mathrm{~Hz}, \mathrm{~J}=1.5,1 \mathrm{H}, \mathrm{H}_{11}\right), 8.21\left(\mathrm{t}, \mathrm{J}=8.2 \mathrm{~Hz}, 1 \mathrm{H}, \mathrm{H}_{12}\right), 8.25\left(\mathrm{~d}, \mathrm{~J}=8.0 \mathrm{~Hz}, 1 \mathrm{H}, \mathrm{H}_{13}\right), 8.98$ $\left(\mathrm{s}, 1 \mathrm{H}, \mathrm{H}_{7}\right), 9.04\left(\mathrm{~s}, 1 \mathrm{H}, \mathrm{H}_{15}\right), 9.23\left(\mathrm{~d}, \mathrm{~J}=7.6 \mathrm{~Hz}, 1 \mathrm{H}, \mathrm{H}_{10}\right) ;{ }^{13} \mathrm{C} \mathrm{NMR}\left(\mathrm{CDCl}_{3}, 75 \mathrm{MHz}\right) \delta$ ppm: $56.83\left(\mathrm{~s}, 2 \mathrm{C}, \mathrm{OCH}_{3}\right), 106.95\left(\mathrm{~s}, 2 \mathrm{C}, \mathrm{C}_{2,6}\right), 123.60\left(\mathrm{~s}, 1 \mathrm{C}, \mathrm{C}_{1}\right), 125.93\left(\mathrm{~s}, 1 \mathrm{C}, \mathrm{C}_{10}\right), 128.30$ $\left(\mathrm{s}, 1 \mathrm{C}, \mathrm{C}_{9}\right), 128.64\left(\mathrm{~s}, 1 \mathrm{C}, \mathrm{C}_{13}\right), 134.14\left(\mathrm{~s}, 1 \mathrm{C}, \mathrm{C}_{11}\right), 136.27\left(\mathrm{~s}, 1 \mathrm{C}, \mathrm{C}_{12}\right), 140.00\left(\mathrm{~s}, 1 \mathrm{C}, \mathrm{C}_{4}\right)$, $145.01\left(\mathrm{~s}, 1 \mathrm{C}, \mathrm{C}_{15}\right), 147.95\left(\mathrm{~s}, 1 \mathrm{C}, \mathrm{C}_{14}\right), 148.66\left(\mathrm{~s}, 2 \mathrm{C}, \mathrm{C}_{3,5}\right), 153.94\left(\mathrm{~s}, 1 \mathrm{C}, \mathrm{C}_{7}\right), 162.24(\mathrm{~s}, 1 \mathrm{C}$, $\mathrm{C}_{8}$ ); UV (EtOH, $\left.50 \mu \mathrm{M}, 25^{\circ} \mathrm{C}\right): \lambda=300 \mathrm{~nm}, \varepsilon=13220 \mathrm{~mol}^{-1} \cdot \mathrm{L}^{\mathrm{cm}} \mathrm{cm}^{-1}, \lambda=372 \mathrm{~nm}, \varepsilon=22180$ mol $^{-1}$.L.cm ${ }^{-1}$; FAB MS: m/z: $325\left[\mathrm{M}+\mathrm{H}^{+}\right]$; ES HRMS: $\left[\mathrm{M}+\mathrm{H}^{+}\right] \mathrm{C}_{17} \mathrm{H}_{17} \mathrm{~N}_{4} \mathrm{O}_{3}: 325.1315$ (calcd 325.1301).

(E)-4-Hydroxy-3,5-dimethoxybenzaldehyde -1,3-benzothiazol-2-ylhydrazone (2)

Yellow solid; yield 97\%; m.p. 219-221 ${ }^{\circ} \mathrm{C}$; IR (KBr) $v \mathrm{~cm}^{-1}: 3543(\mathrm{O}-\mathrm{H}) ; 3480(\mathrm{~N}-\mathrm{H}) ; 3185$ (=C-H ethyl.), 3068 (C-H arom.), 2846 (C-H, O-CH 3$), 1672$ (C=N ethyl.), 1608 (C=C arom.), $1510\left(\mathrm{C}=\mathrm{C}\right.$ arom.), 1249 (O-C arom), 1219 (O-C), 1110 (O-C); ${ }^{1} \mathrm{H}$ NMR $\left(\mathrm{CDCl}_{3}, 300 \mathrm{MHz}\right)$ 
$\delta$ ppm : $3.97\left(\mathrm{~s}, 6 \mathrm{H}, \mathrm{OCH}_{3}\right) ; 6.94\left(\mathrm{~s}, 2 \mathrm{H}, \mathrm{H}_{2,6}\right) ; 7.18\left(\mathrm{t}, 1 \mathrm{H}, \mathrm{J}=7.8 \mathrm{~Hz}, \mathrm{H}_{5^{\prime}}\right) ; 7.36(\mathrm{t}, 1 \mathrm{H}, \mathrm{J}=$ $\left.8.1 \mathrm{~Hz}, \mathrm{H}_{11}\right) ; 7.56\left(\mathrm{~d}, 1 \mathrm{H}, \mathrm{J}=8.1 \mathrm{~Hz}, \mathrm{H}_{10}\right) ; 7.68\left(\mathrm{~d}, 1 \mathrm{H}, \mathrm{J}=7.8 \mathrm{~Hz}, \mathrm{H}_{13}\right) ; 7.90\left(\mathrm{~s}, 1 \mathrm{H}, \mathrm{H}_{7}\right) .{ }^{13} \mathrm{C}$ NMR $\left(\mathrm{CDCl}_{3}, 75 \mathrm{MHz}\right) \delta \mathrm{ppm}: 56.45\left(\mathrm{~s}, 2 \mathrm{C}, \mathrm{OCH}_{3}\right) ; 104.67$ (s, 2C, $\left.\mathrm{C}_{2,6}\right) ; 121,97$ (s, 2C, $\left.\mathrm{C}_{11}\right) ; 122.06\left(\mathrm{~s}, 1 \mathrm{C}, \mathrm{C}_{12}\right) ; 125.05\left(\mathrm{~s}, 1 \mathrm{C}, \mathrm{C}_{1}\right) ; 126.44\left(\mathrm{~s}, 2 \mathrm{C}, \mathrm{C}_{10,13}\right) ; 129.43\left(\mathrm{~s}, 2 \mathrm{C}, \mathrm{C}_{14}\right) ; 129.44$ $\left(\mathrm{s}, 1 \mathrm{C}, \mathrm{C}_{9}\right) ; 138.05\left(\mathrm{~s}, 1 \mathrm{C}, \mathrm{C}_{4}\right) ; 145.46\left(\mathrm{~s}, 1 \mathrm{C}, \mathrm{C}_{7}\right) ; 148.63\left(\mathrm{~s}, 2 \mathrm{C}, \mathrm{C}_{3,5}\right) ; 167.24\left(\mathrm{~s}, 1 \mathrm{C}, \mathrm{C}_{8}\right)$. UV $\left(\mathrm{EtOH}, 29.92 \mu \mathrm{M}, 25^{\circ} \mathrm{C}\right): \lambda=342 \mathrm{~nm}, \varepsilon=34920 \mathrm{~mol}^{-1} . \mathrm{L} \cdot \mathrm{cm}^{-1}$; ES MS: m/z $330.4\left[\mathrm{M}+\mathrm{H}^{+}\right]$; ES HRMS: $\left[\mathrm{M}+\mathrm{H}^{+}\right] \mathrm{C}_{16} \mathrm{H}_{16} \mathrm{~N}_{3} \mathrm{O}_{3} \mathrm{~S}: 330.0927$ (calcd 330.0912).

(E)-4-Hydroxy-3,5-dimethoxybenzaldehyde(2-fluorophenyl)hydrazone hydrocloride (3) Green solid; yield 82\%; m.p. 104-106 ${ }^{\circ} \mathrm{C}$; IR (KBr) $v \mathrm{~cm}^{-1}: 3422(\mathrm{~N}-\mathrm{H}), 3332(\mathrm{O}-\mathrm{H}), 3243$ (=C-H ethyl.), 3009 (C-H arom.), 2938 (C-H, O-CH 3$), 1619$ (C=N ethyl.), $1591(\mathrm{C}=\mathrm{C}$ arom.), 1514 (C=C arom.), 1243 (O-C arom), 1206 (O-C), 1115 (C-F); ${ }^{1} \mathrm{H}$ NMR (CD $\left.{ }_{3} \mathrm{OD}, 300 \mathrm{MHz}\right)$ $\delta$ ppm: $3.80\left(\mathrm{~s}, 6 \mathrm{H}, \mathrm{OCH}_{3}\right), 6.62(\mathrm{dddd}, \mathrm{J}=8.1 \mathrm{~Hz}, \mathrm{~J}=7.4 \mathrm{~Hz}, \mathrm{~J}=4.8 \mathrm{~Hz}, \mathrm{~J}=1.6 \mathrm{~Hz}, 1 \mathrm{H}$, $\left.\mathrm{H}_{4^{\prime}}\right), 6.88\left(\mathrm{~s}, 2 \mathrm{H}, \mathrm{H}_{2,6}\right), 6.89\left(\mathrm{ddd}, \mathrm{J}=8.4 \mathrm{~Hz}, \mathrm{~J}=4.1 \mathrm{~Hz}, \mathrm{~J}=1.3 \mathrm{~Hz}, 1 \mathrm{H}, \mathrm{H}_{6^{\prime}}\right), 6.96(\mathrm{dddd}, \mathrm{J}=$ $\left.8.1 \mathrm{~Hz}, \mathrm{~J}=7.4 \mathrm{~Hz}, \mathrm{~J}=1.3 \mathrm{~Hz}, \mathrm{~J}=1.3 \mathrm{~Hz}, 1 \mathrm{H}, \mathrm{H}_{5^{\prime}}\right), 7.42(\mathrm{ddd}, \mathrm{J}=8.4 \mathrm{~Hz}, \mathrm{~J}=8.3 \mathrm{~Hz}, \mathrm{~J}=1.6 \mathrm{~Hz}$, $\left.1 \mathrm{H}, \mathrm{H}_{3}\right) 7.80\left(\mathrm{~s}, 1 \mathrm{H}, \mathrm{H}_{7}\right) ;{ }^{13} \mathrm{C} \mathrm{NMR}\left(\mathrm{CD}_{3} \mathrm{OD}, 75 \mathrm{MHz}\right) \delta \mathrm{ppm}: 55.37\left(\mathrm{~s}, 2 \mathrm{C}, \mathrm{OCH}_{3}\right), 103.36$ $\left(\mathrm{s}, 2 \mathrm{C}, \mathrm{C}_{2,6}\right), 113.74\left(\mathrm{~d}, \mathrm{~J}=3.1 \mathrm{~Hz}, 1 \mathrm{C}, \mathrm{C}_{13}\right), 114.18\left(\mathrm{~d}, \mathrm{~J}=180.0 \mathrm{~Hz}, 1 \mathrm{C}, \mathrm{C}_{10}\right), 118.09$ (d, J = $\left.6.8 \mathrm{~Hz}, 1 \mathrm{C}, \mathrm{C}_{11}\right), 124.35\left(\mathrm{~d}, 1 \mathrm{C}, \mathrm{C}_{12}\right), 126.59\left(\mathrm{~s}, 1 \mathrm{C}, \mathrm{C}_{1}\right), 133.90\left(\mathrm{~d}, \mathrm{~J}=9.3 \mathrm{~Hz}, 1 \mathrm{C}, \mathrm{C}_{8}\right), 136,51$ (s, 1C, $\mathrm{C}_{4}$ ), 139.99 (s, 1C, $\mathrm{C}_{7}$ ), 148.07 (s, 2C, $\left.\mathrm{C}_{3,5}\right), 149.70$ (d, J = $\left.237 \mathrm{~Hz}, 1 \mathrm{C}, \mathrm{C}_{9}\right) ;{ }^{19} \mathrm{~F} \mathrm{NMR}$ $\left(\mathrm{CD}_{3} \mathrm{OD}, 282 \mathrm{MHz}\right) \square \mathrm{ppm}:-137.96(\mathrm{~s}, 1 \mathrm{~F}) ; \mathrm{UV}\left(\mathrm{EtOH}, 45.24 \mu \mathrm{M}, 25^{\circ} \mathrm{C}\right): \lambda=244 \mathrm{~nm}, \varepsilon=$ $16220 \mathrm{~mol}^{-1} \cdot \mathrm{L}^{\mathrm{cm}}{ }^{-1}, \lambda=290 \mathrm{~nm}, \varepsilon=9750 \mathrm{~mol}^{-1} \cdot \mathrm{L}^{\mathrm{c}} \mathrm{cm}^{-1}, \lambda=345 \mathrm{~nm}, \varepsilon=22700 \mathrm{~mol}^{-1} \cdot \mathrm{L} \cdot \mathrm{cm}^{-1}$; ES MS: m/z 291.2 [M+H'] ${ }^{+}$; ES HRMS: $\left[\mathrm{M}+\mathrm{H}^{+}\right] \mathrm{C}_{15} \mathrm{H}_{16} \mathrm{~N}_{2} \mathrm{O}_{3} \mathrm{~F}: 291.1135$ (calcd 291.1145).

(E)-4-Hydroxy-3,5-dimethoxybenzaldehyde(4-fluorophenyl)hydrazone hydrocloride (4) Brown crystals; yield 47\%; m.p. 170-174 ${ }^{\circ} \mathrm{C}$; IR (KBr) $v \mathrm{~cm}^{-1}: 3493(\mathrm{O}-\mathrm{H}), 1611(\mathrm{C}=\mathrm{N})$, 
$1584(\mathrm{C}=\mathrm{C}), 1505\left(\mathrm{C}=\mathrm{C}\right.$ arom.), $1212(\mathrm{O}-\mathrm{C}), 1117(\mathrm{C}-\mathrm{F}) ;{ }^{1} \mathrm{H}$ NMR $\left(\mathrm{CD}_{3} \mathrm{OD}, 300 \mathrm{MHz}\right) \delta$ ppm: $3.90\left(\mathrm{~s}, 6 \mathrm{H}, \mathrm{OCH}_{3}\right), 6.95\left(\mathrm{~s}, 2 \mathrm{H}, \mathrm{H}_{2,6}\right), 6.96\left(\mathrm{dd}, \mathrm{J}=9.0 \mathrm{~Hz}, 2 \mathrm{H}, \mathrm{H}_{3^{\prime}, 5^{\prime}}\right), 7.07(\mathrm{dd}, \mathrm{J}=$ 9.1Hz, J = 4.8 Hz, 2H, $\left.\mathrm{H}_{2^{\prime}, 6^{\prime}}\right), 7.70\left(\mathrm{~s}, 1 \mathrm{H}, \mathrm{H}_{7}\right) ;{ }^{13} \mathrm{C}$ NMR $\left(\mathrm{CD}_{3} \mathrm{OD}, 75 \mathrm{MHz}\right) \delta \mathrm{ppm}: 55.32(\mathrm{~s}$, 2C, $\left.\mathrm{OCH}_{3}\right), 102.92\left(\mathrm{~s}, 2 \mathrm{C}, \mathrm{C}_{2,6}\right), 112.71\left(\mathrm{~d}, \mathrm{~J}=7.5 \mathrm{~Hz}, 2 \mathrm{C}, \mathrm{C}_{9,13}\right), 114.93$ (d, J=22.5 Hz, 2C, $\left.\mathrm{C}_{10,12}\right), 127.19$ (s, 1C, $\mathrm{C}_{1}$ ), 137.52 (s, 1C, $\mathrm{C}_{7}$ ), 141.13 (d, J=209.1 Hz, 1C, $\mathrm{C}_{11}$ ), 142.41 (s, 1C, $\left.\mathrm{C}_{4}\right), 148.06\left(\mathrm{~s}, 2 \mathrm{C}, \mathrm{C}_{3,5}\right), 158.17\left(\mathrm{~s}, 1 \mathrm{C}, \mathrm{C}_{8}\right) ;{ }^{19} \mathrm{~F}$ NMR $\left(\mathrm{CD}_{3} \mathrm{OD}, 282 \mathrm{MHz}\right) \delta \mathrm{ppm}:-128.77$ (s, 1F); UV (EtOH, $\left.39.73 \mu \mathrm{M}, 25^{\circ} \mathrm{C}\right): \lambda=240 \mathrm{~nm}, \varepsilon=17940 \mathrm{~mol}^{-1} \cdot \mathrm{L}^{\mathrm{c}} \mathrm{cm}^{-1}, \lambda=313 \mathrm{~nm}, \varepsilon=$ $19680 \mathrm{~mol}^{-1} \cdot \mathrm{L} \cdot \mathrm{cm}^{-1}, \lambda=345 \mathrm{~nm}, \varepsilon=25370 \mathrm{~mol}^{-1} \cdot \mathrm{L} \cdot \mathrm{cm}^{-1} \mathrm{ES} \mathrm{MS}: \mathrm{m} / \mathrm{z} 291.1\left[\mathrm{M}+\mathrm{H}^{+}\right]$; ES HRMS: [M+H $\left.{ }^{+}\right] \mathrm{C}_{10} \mathrm{H}_{15} \mathrm{~N}_{3} \mathrm{O}_{3}: 291.1118$ (calcd 291.1107).

(E)-4-Hydroxy-3,5-dimethoxybenzaldehyde[2,6-dichloro-4-(trifluoromethyl)phenyl] hydrazone (5) White crystals; yield 48\%. m.p. 161-163을 $\mathrm{IR}(\mathrm{KBr}) \vee \mathrm{cm}^{-1}: 3430(\mathrm{~N}-\mathrm{H})$, 3295 (O-H), 3080 (=C-H ethyl.), 3007 (C-H arom.), 2945 (C-H, O-CH 3 ), 1607 (C=N ethyl.), $1587(\mathrm{C}=\mathrm{C}$ arom.), 1514 (C=C arom.), 1261 (O-C arom), 1209 (O-C), 1116 (C-F), 1104 (C$\mathrm{Cl}) ;{ }^{1} \mathrm{H}$ NMR $\left(\mathrm{CD}_{3} \mathrm{OD}, 300 \mathrm{MHz}\right) \delta$ ppm: 3.89 (s, 6H, $\left.\mathrm{OCH}_{3}\right), 7.01$ (s, 2H, $\left.\mathrm{H}_{2,6}\right), 7.64(\mathrm{~s}, 2 \mathrm{H}$, $\left.\mathrm{H}_{3^{\prime}, 5^{\prime}}\right), 7.97\left(\mathrm{~s}, 1 \mathrm{H}, \mathrm{H}_{7}\right) ;{ }^{13} \mathrm{C} \mathrm{NMR}\left(\mathrm{CD}_{3} \mathrm{OD}, 75 \mathrm{MHz}\right) \delta \mathrm{ppm}: 55.32\left(\mathrm{~s}, 2 \mathrm{C}, \mathrm{OCH}_{3}\right), 103.52(\mathrm{~s}$, 2C, $\left.\mathrm{C}_{2,6}\right), 123.09$ (q, J = $\left.34 \mathrm{~Hz}, 1 \mathrm{C}, \mathrm{C}_{11}\right), 123.20$ (q, J = 270.9 Hz, 1C, $\left.\underline{\mathrm{CF}}_{3}\right), 123.87$ (s, 2C, $\left.\mathrm{C}_{9,13}\right), 126.15\left(\mathrm{~s}, 1 \mathrm{C}, \mathrm{C}_{1}\right), 126.17\left(\mathrm{q}, \mathrm{J}=3.5 \mathrm{~Hz}, 2 \mathrm{C}, \mathrm{C}_{10,12}\right), 136.91\left(\mathrm{~s}, 1 \mathrm{C}, \mathrm{C}_{4}\right), 141.55(\mathrm{~s}, 1 \mathrm{C}$, $\left.\mathrm{C}_{8}\right), 143.15\left(\mathrm{~s}, 1 \mathrm{C}, \mathrm{C}_{7}\right), 148.05\left(\mathrm{~s}, 2 \mathrm{C}, \mathrm{C}_{3,5}\right) .{ }^{19} \mathrm{~F}$ NMR $\left(\mathrm{CD}_{3} \mathrm{OD}, 282 \mathrm{MHz}\right) \delta \mathrm{ppm}$ : -63.55 (s, 1F); UV (EtOH, $\left.56.25 \mu \mathrm{M}, 25^{\circ} \mathrm{C}\right): \lambda=273 \mathrm{~nm}, \varepsilon=6860 \mathrm{~mol}^{-1} \cdot \mathrm{L}^{\mathrm{c}} \mathrm{cm}^{-1}, \lambda=340 \mathrm{~nm}, \varepsilon=19270$

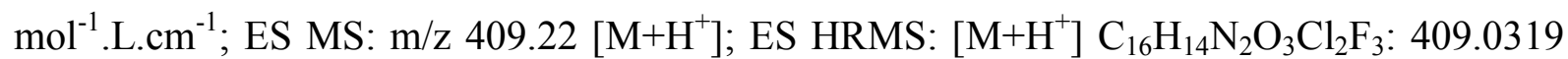
(calcd 409.0334).

(E)-2-(4-Hydroxy-3,5-dimethoxybenzylidene)hydrazinecarboximidamide hydrochloride (6) Yellow solid; yield 86\%; m.p. 226-228 ${ }^{\circ} \mathrm{C}$; IR $(\mathrm{KBr}) \vee \mathrm{cm}^{-1}$ : $3387(\mathrm{O}-\mathrm{H})$, 
3320 (N-H), 3200 (=C-H ethyl.), 3094 (C-H arom.), 2967 (C-H, O-CH 3 ), 1689 (C=N ethyl.), $1609(\mathrm{C}=\mathrm{C}$ arom. $), 1519(\mathrm{C}=\mathrm{C}$ arom. $), 1469(\mathrm{~N}-\mathrm{H}), 1115(\mathrm{O}-\mathrm{C}$ arom $) ;{ }^{1} \mathrm{H}$ NMR $\left(\mathrm{CD}_{3} \mathrm{OD}, 300\right.$ $\mathrm{MHz}) \delta \mathrm{ppm}: 3.92\left(\mathrm{~s}, 6 \mathrm{H}, \mathrm{OCH}_{3}\right), 7.14\left(\mathrm{~s}, 2 \mathrm{H}, \mathrm{H}_{2,6}\right), 8.00\left(\mathrm{~s}, 1 \mathrm{H}, \mathrm{H}_{7}\right),{ }^{13} \mathrm{C} \mathrm{NMR}\left(\mathrm{CD}_{3} \mathrm{OD}, 75\right.$ $\mathrm{MHz}) \delta \mathrm{ppm}: 55.53\left(\mathrm{~s}, 2 \mathrm{C}, \mathrm{OCH}_{3}\right), 105.00\left(\mathrm{~s}, 2 \mathrm{C}, \mathrm{C}_{2,6}\right), 124.03\left(\mathrm{~s}, 1 \mathrm{C}, \mathrm{C}_{1}\right), 137.25(\mathrm{~s}, 1 \mathrm{C}$, $\left.\mathrm{C}_{4}\right), 148.15\left(\mathrm{~s}, 2 \mathrm{C}, \mathrm{C}_{3,5}\right), 148.52\left(\mathrm{~s}, 2 \mathrm{C}, \mathrm{C}_{7}\right), 208.7\left(\mathrm{~s}, 1 \mathrm{C}, \mathrm{C}_{8}\right)$; UV (EtOH, $\left.50 \mu \mathrm{M}, 25^{\circ} \mathrm{C}\right): \lambda=$ $234 \mathrm{~nm}, \varepsilon=19080 \mathrm{~mol}^{-1} \cdot \mathrm{L} \cdot \mathrm{cm}^{-1}, \lambda=315 \mathrm{~nm}, \varepsilon=19160 \mathrm{~mol}^{-1} \cdot \mathrm{L} \cdot \mathrm{cm}^{-1} ;$ ES MS: m/z 239.2 $\left[\mathrm{M}+\mathrm{H}^{+}\right]$; ES HRMS: $\left[\mathrm{M}+\mathrm{H}^{+}\right] \mathrm{C}_{10} \mathrm{H}_{15} \mathrm{~N}_{3} \mathrm{O}_{3}: 239.1115$ (calcd 239.1144).

(E)-N'-(4-Hydroxy-3,5-dimethoxybenzylidene)nicotinohydrazide (7) Yellow solid; yield 89 \%; m.p. $214-216{ }^{\circ} \mathrm{C}$; IR (KBr) $\vee$ cm$^{-1}: 3480(\mathrm{O}-\mathrm{H}), 3185$ (=C-H ethyl.), 2995 (C-H arom.), $2848\left(\mathrm{C}-\mathrm{H}, \mathrm{O}-\mathrm{CH}_{3}\right), 1645(\mathrm{C}=\mathrm{N}$ ethyl. $), 1600(\mathrm{C}=\mathrm{C}$ arom. $), 1580(\mathrm{~N}-\mathrm{H}), 1510(\mathrm{C}=\mathrm{C}$ arom.), 1252 (O-C arom) , 1214 (O-C); ${ }^{1} \mathrm{H}$ NMR (CD $\left.{ }_{3} \mathrm{OD}, 300 \mathrm{MHz}\right) \delta$ ppm: 3.92 (s, 6H, $\left.\mathrm{OCH}_{3}\right), 7.20\left(\mathrm{~s}, 2 \mathrm{H}, \mathrm{H}_{2,6}\right), 7.60\left(\mathrm{dd}, 1 \mathrm{H}, \mathrm{J}=7.7 \mathrm{~Hz}, \mathrm{~J}=4.6 \mathrm{~Hz}, \mathrm{H}_{11}\right), 8.23\left(\mathrm{~s}, 1 \mathrm{H}, \mathrm{H}_{7}\right), 8.36(\mathrm{~d}$, $\left.\mathrm{J}=7.8 \mathrm{~Hz}, 1 \mathrm{H}, \mathrm{H}_{12}\right), 8.74\left(\mathrm{dd}, \mathrm{J}=1.1 \mathrm{~Hz}, \mathrm{~J}=4.8 \mathrm{~Hz}, 1 \mathrm{H}, \mathrm{H}_{10}\right), 9.09\left(\mathrm{~d}, \mathrm{~J}=1.2 \mathrm{~Hz}, 1 \mathrm{H}, \mathrm{H}_{13}\right)$. ${ }^{13} \mathrm{C}$ NMR $\left(\mathrm{CD}_{3} \mathrm{OD}, 75 \mathrm{MHz}\right) \delta \mathrm{ppm}: 55.43\left(\mathrm{~s}, 2 \mathrm{C}, \mathrm{OCH}_{3}\right), 105.05\left(\mathrm{~s}, 2 \mathrm{C}, \mathrm{C}_{2,6}\right), 123.84(\mathrm{~s}, 1 \mathrm{C}$, $\left.\mathrm{C}_{10}\right), 124.62\left(\mathrm{~s}, 1 \mathrm{C}, \mathrm{C}_{1}\right), 129.40\left(\mathrm{~s}, 1 \mathrm{C}, \mathrm{C}_{8}\right), 136.00\left(\mathrm{~s}, 1 \mathrm{C}, \mathrm{C}_{9}\right), 138.36\left(\mathrm{~s}, 1 \mathrm{C}, \mathrm{C}_{4}\right), 147.94(\mathrm{~s}$, 2C, $\mathrm{C}_{12}$ ), 148.09 (s, 2C, $\left.\mathrm{C}_{3,5}\right), 150.36\left(\mathrm{~s}, 1 \mathrm{C}, \mathrm{C}_{7}\right), 151.69$ (s,1C, $\left.\mathrm{C}_{11}\right), 163.12$ (s, 1C, CO); UV $\left(\mathrm{EtOH}, 49.76 \mu \mathrm{M}, 25^{\circ} \mathrm{C}\right): \lambda=330 \mathrm{~nm}, \varepsilon=22080 \mathrm{~mol}^{-1} . \mathrm{L} \cdot \mathrm{cm}^{-1}$; ES MS: m/z $302.2\left[\mathrm{M}+\mathrm{H}^{+}\right]$; ES HRMS: $\left[\mathrm{M}+\mathrm{H}^{+}\right] \mathrm{C}_{15} \mathrm{H}_{16} \mathrm{~N}_{3} \mathrm{O}_{4}: 302.1143$ (calcd 302.1141).

(E)-N'-(4-Hydroxy-3,5-dimethoxybenzylidene)isonicotinohydrazide (8) Yellow solid; yield 97\%; m.p. 195-198 ${ }^{\circ} \mathrm{C}$; IR (KBr) v cm${ }^{-1}$ : 3397 (O-H), 3195 (=C-H ethyl.), 2846 (C-H, O- $\left.\mathrm{CH}_{3}\right), 1646(\mathrm{C}=\mathrm{N}$ ethyl. $), 1587(\mathrm{C}=\mathrm{C}$ arom. $), 1515(\mathrm{C}=\mathrm{C}$ arom. $), 1453$ ( $\mathrm{C}=\mathrm{C}$ arom. $), 1219$ (C-O), $1116(\mathrm{C}-\mathrm{O}) ;{ }^{1} \mathrm{H}$ NMR (DMSO-d $\left.6,300 \mathrm{MHz}\right) \delta \mathrm{ppm}: 3.80\left(\mathrm{~s}, 6 \mathrm{H}, \mathrm{OCH}_{3}\right), 7.06(\mathrm{~s}, 2 \mathrm{H}$, $\left.\mathrm{H}_{2,6}\right), 7.78\left(\mathrm{dd}, \mathrm{J}=4.5 \mathrm{~Hz}, \mathrm{~J}=1.7 \mathrm{~Hz}, 2 \mathrm{H}, \mathrm{H}_{10,13}\right), 8.29\left(\mathrm{~s}, 1 \mathrm{H}, \mathrm{H}_{7}\right), 8.74(\mathrm{dd}, \mathrm{J}=4.5 \mathrm{~Hz}, \mathrm{~J}=$ 
$\left.1.7 \mathrm{~Hz}, 2 \mathrm{H}, \mathrm{H}_{11,12}\right) ;{ }^{13} \mathrm{C} \mathrm{NMR}\left(\mathrm{DMSO}-d_{6}, 75 \mathrm{MHz}\right) \delta \mathrm{ppm}: 56.47\left(\mathrm{~s}, 2 \mathrm{C}, \mathrm{OCH}_{3}\right), 105.32(\mathrm{~s}$, 2C, $\left.\mathrm{C}_{2,6}\right), 122.02\left(\mathrm{~s}, 2 \mathrm{C}, \mathrm{C}_{10,13}\right), 124.65\left(\mathrm{~s}, 1 \mathrm{C}, \mathrm{C}_{1}\right), 138.39\left(\mathrm{~s}, 1 \mathrm{C}, \mathrm{C}_{4}\right), 140.96\left(\mathrm{~s}, 1 \mathrm{C}, \mathrm{C}_{9}\right)$, $148.44\left(\mathrm{~s}, 2 \mathrm{C}, \mathrm{C}_{3,5}\right), 150.45\left(\mathrm{~s}, 1 \mathrm{C}, \mathrm{C}_{7}\right), 150.67\left(\mathrm{~s}, 2 \mathrm{C}, \mathrm{C}_{11,12}\right), 162.24\left(\mathrm{~s}, 1 \mathrm{C}, \mathrm{C}_{8}\right)$; UV (EtOH, $\left.49.66 \mu \mathrm{M}, 25^{\circ} \mathrm{C}\right): \lambda=333 \mathrm{~nm}, \varepsilon=20960 \mathrm{~mol}^{-1} \cdot \mathrm{L} \cdot \mathrm{cm}^{-1}$; FAB MS: m/z $302\left[\mathrm{M}+\mathrm{H}^{+}\right]$; ES HRMS: $\left[\mathrm{M}+\mathrm{H}^{+}\right] \mathrm{C}_{15} \mathrm{H}_{16} \mathrm{~N}_{3} \mathrm{O}_{4}: 302.1148$ (calcd 302.1141).

(E)- $N^{\prime}$-(4-Hydroxy-3,5-dimethoxybenzylidene)-3-methyl-1H-pyrazole-5-

carbohydrazide (9) Yellow solid; yield 64\%; m.p. 223-225 ${ }^{\circ} \mathrm{C}$; IR (KBr) $v \mathrm{~cm}^{-1}: 3433(\mathrm{~N}-\mathrm{H})$, 3293 (O-H), 3208 (=C-H ethyl.), 3117 (C-H arom.), 2964 (C-H, O-CH3), 1664 (C=O ethyl.), $1621(\mathrm{C}=\mathrm{N}$ ethyl. $), 1590(\mathrm{C}=\mathrm{C}$ arom. $), 1516(\mathrm{C}=\mathrm{C}$ arom. $), 1327(\mathrm{C}-\mathrm{N}), 1241(\mathrm{O}-\mathrm{C}$ arom $)$, $1216(\mathrm{O}-\mathrm{C}) ;{ }^{1} \mathrm{H}$ NMR $\left(\mathrm{CD}_{3} \mathrm{OD}, 300 \mathrm{MHz}\right) \delta \mathrm{ppm}: 2.36\left(\mathrm{~s}, 3 \mathrm{H}, \mathrm{CH}_{3}\right), 3.92\left(\mathrm{~s}, 6 \mathrm{H}, \mathrm{OCH}_{3}\right)$, $6.62\left(\mathrm{~s}, 1 \mathrm{H}, \mathrm{H}_{10}\right), 7.21\left(\mathrm{~s}, 2 \mathrm{H}, \mathrm{H}_{2,6}\right), 8.20\left(\mathrm{~s}, 1 \mathrm{H}, \mathrm{H}_{7}\right) ;{ }^{13} \mathrm{C} \mathrm{NMR}\left(\mathrm{CD}_{3} \mathrm{OD}, 75 \mathrm{MHz}\right) \delta \mathrm{ppm}:$ $10.80\left(\mathrm{~s}, 1 \mathrm{C}, \mathrm{CH}_{3}\right), 56.49\left(\mathrm{~s}, 2 \mathrm{C}, \mathrm{OCH}_{3}\right), 104.95\left(\mathrm{~s}, 2 \mathrm{C}, \mathrm{C}_{2,6}\right), 105.27\left(\mathrm{~s}, 1 \mathrm{C}, \mathrm{C}_{10}\right), 125.32$ (s, $\left.1 \mathrm{C}, \mathrm{C}_{1}\right), 138.22\left(\mathrm{~s}, 1 \mathrm{C}, \mathrm{C}_{4}\right), 140.46\left(\mathrm{~s}, 1 \mathrm{C}, \mathrm{C}_{11}\right), 146.49\left(\mathrm{~s}, \mathrm{C}, \mathrm{C}_{7}\right), 148.29\left(\mathrm{~s}, 2 \mathrm{C}, \mathrm{C}_{3,5}\right), 148.59$ $\left(\mathrm{s}, 1 \mathrm{C}, \mathrm{C}_{9}\right), 158.70\left(\mathrm{~s}, 1 \mathrm{C}, \mathrm{C}_{8}\right)$; UV (EtOH, $\left.40 \mu \mathrm{M}, 25^{\circ} \mathrm{C}\right): \lambda=240 \mathrm{~nm}, \varepsilon=18430 \mathrm{~mol}^{-1} .{\mathrm{L} . \mathrm{cm}^{-}}^{-}$ ${ }^{1}, \lambda=327 \mathrm{~nm}, \varepsilon=27500 \mathrm{~mol}^{-1} \cdot \mathrm{L} \cdot \mathrm{cm}^{-1}$; ES MS: m/z $305.2\left[\mathrm{M}+\mathrm{H}^{+}\right]$; ES HRMS: $\left[\mathrm{M}+\mathrm{H}^{+}\right]$ $\mathrm{C}_{14} \mathrm{H}_{17} \mathrm{~N}_{4} \mathrm{O}_{4}: 305.1252$ (calcd 305.1250).

(E)-Benzyl-2-(4-hydroxy-3,5-dimethoxybenzylidene)hydrazinecarboxylate (10)

Yellow solid; yield 90\%; m.p. 115-117 C; IR (KBr) $v \mathrm{~cm}^{-1}: 3350(\mathrm{O}-\mathrm{H}), 3243$ (=C-H ethyl.), 3071 (C-H arom.), $2964\left(\mathrm{C}-\mathrm{H}, \mathrm{O}-\mathrm{CH}_{3}\right), 1707(\mathrm{C}=\mathrm{O}), 1673(\mathrm{C}=\mathrm{N}$ ethyl.), $1588(\mathrm{C}=\mathrm{C}$ arom.), 1548 (N-H), 1513 (C=C arom.), 1245 (O-C arom), 1217 (O-C); ${ }^{1} \mathrm{H}$ NMR ( $\mathrm{CD}_{3} \mathrm{OD}$, $300 \mathrm{MHz}) \delta \mathrm{ppm}: 3.77\left(\mathrm{~s}, 6 \mathrm{H}, \mathrm{OCH}_{3}\right), 5.13\left(\mathrm{~s}, 2 \mathrm{H}, \mathrm{H}_{9}\right), 6.95\left(\mathrm{~s}, 2 \mathrm{H}, \mathrm{H}_{2,6}\right), 7.29\left(\mathrm{~m}, 5 \mathrm{H}, \mathrm{H}_{\mathrm{ar}}\right)$, $7.74\left(\mathrm{~s}, 1 \mathrm{H}, \mathrm{H}_{7}\right) ;{ }^{13} \mathrm{C} \mathrm{NMR}\left(\mathrm{CD}_{3} \mathrm{OD}, 75 \mathrm{MHz}\right) \delta \mathrm{ppm}: 55.40\left(\mathrm{~s}, 2 \mathrm{C}, \mathrm{OCH}_{3}\right), 66.66\left(\mathrm{~s}, 2 \mathrm{C}, \mathrm{C}_{9}\right)$, $104.34\left(\mathrm{~s}, 2 \mathrm{C}, \mathrm{C}_{2,6}\right), 125.17\left(\mathrm{~s}, 1 \mathrm{C},\left(\mathrm{s}, 1 \mathrm{C}, \mathrm{C}_{1}\right), 127.17\left(\mathrm{~s}, 1 \mathrm{C}, \mathrm{C}_{\mathrm{ar}}\right), 127.72\left(\mathrm{~s}, 2 \mathrm{C}, \mathrm{C}_{\mathrm{ar}}\right), 127.86\right.$ 
$\left(\mathrm{s}, 2 \mathrm{C}, \mathrm{C}_{\mathrm{ar}}\right), 136.47\left(\mathrm{~s}, 1 \mathrm{C}, \mathrm{C}_{4}\right), 137.50\left(\mathrm{~s}, 1 \mathrm{C}, \mathrm{C}_{10}\right), 145.78\left(\mathrm{~s}, 1 \mathrm{C}, \mathrm{C}_{7}\right), 148.36\left(\mathrm{~s}, 2 \mathrm{C}, \mathrm{C}_{3,5}\right)$, $155.10(\mathrm{~s}, 1 \mathrm{C}, \mathrm{CO}) ; \mathrm{UV}\left(\mathrm{EtOH}, 45.24 \mu \mathrm{M}, 25^{\circ} \mathrm{C}\right): \lambda=233 \mathrm{~nm}, \varepsilon=25040 \mathrm{~mol}^{-1} \cdot \mathrm{L}^{\mathrm{cm}} \mathrm{cm}^{-1} ; \lambda=$ $308 \mathrm{~nm}, \varepsilon=23080 \mathrm{~mol}^{-1} \cdot \mathrm{L} \cdot \mathrm{cm}^{-1} ;$ ES MS: m/z $331.2\left[\mathrm{M}+\mathrm{H}^{+}\right]$; ES HRMS: $\left[\mathrm{M}+\mathrm{H}^{+}\right]$ $\mathrm{C}_{17} \mathrm{H}_{19} \mathrm{~N}_{2} \mathrm{O}_{5}: 331.1281$ (calcd 331.1294).

\subsection{DPPH scavenging activity}

The $\mathrm{DPPH}^{*}$ solution has a deep violet colour. Radical scavenging activity of antioxidant compounds can be measured spectrophotometrically at $517 \mathrm{~nm}$. The DPPH radical shows absorbance at $517 \mathrm{~nm}$, that decreases by the bleaching of a violet coloured ethanol solution upon reduction by an antioxidant or a radical through hydrogen atom or electron transfer to the odd electron in $\mathrm{DPPH}^{\circ}$ producing the pale yellow non-radical form (DPPH-H). This decrease is proportional to the increase of non-radical forms of DPPH [22]. Briefly, $6 \mathrm{mM}$ solution of $\mathrm{DPPH}^{*}$ was prepared in ethanol and, $90 \mu \mathrm{L}$ of this solution was added to $2.1 \mathrm{~mL}$ of hydrazones solution in ethanol at different concentrations $(5-500 \mu \mathrm{M})$. The absorbance was measured at $\mathrm{t}=0$ min $\left(\mathrm{A}_{0}\right)$ and after 60 min incubation at room temperature $\left(\mathrm{A}_{60}\right)$. Percentage inhibition of DPPH free radical (I\%) was calculated in the following way: $\mathrm{I} \%=$ $100 *\left(\mathrm{~A}_{0}-\mathrm{A}_{60}\right) / \mathrm{A}_{0}$. IC50 represents concentration of the tested compound, providing $50 \%$ inhibition of DPPH radicals. All experiments were carried out in triplicate. The 6-hydroxyl2,5,7,8-tetramethylchroman-2-carboxylic acid (Trolox) was used as antioxidant reference [14].

\subsection{ABTS $^{\bullet+}$ scavenging activity}

The second test is based on the ability of antiradical molecules to quench the $\mathrm{ABTS}^{\circ+}$, a bluegreen chromophore with characteristic absorption at $734 \mathrm{~nm}$. The addition of antioxidants to the preformed radical cation reduces it to ABTS, determining a decolourization. In this 
method, an antioxidant is added to a pre-formed ABTS radical solution, and after a fixed time period, the remaining $\mathrm{ABTS}^{\circ+}$ is quantified spectrophotometrically at $734 \mathrm{~nm}$ [22]. The ABTS $^{\circ+}$ was produced by reacting $7 \mathrm{mM}$ ABTS in $\mathrm{H}_{2} \mathrm{O}$ with $2.45 \mathrm{mM}$ potassium persulfate $\left(\mathrm{K}_{2} \mathrm{~S}_{2} \mathrm{O}_{8}\right)$, stored in the dark at room temperature for $12-16 \mathrm{~h}$ before use (the radical was stable for more than two days under these conditions). The $\mathrm{ABTS}^{\circ+}$ solution was further diluted in ethanol until the initial absorbance value of $0.70 \pm 0.02$ at $734 \mathrm{~nm}$ at $30^{\circ} \mathrm{C}$. Then, in a $3 \mathrm{~mL}$ solution of $\mathrm{ABTS}^{\cdot+}$, was added an ethanolic solution of the compound under study so that their final concentrations vary between 0-15 $\mu \mathrm{M}$. In each case, a 20-80\% decrease in the initial absorbance of the reaction solution was achieved.

The absorbance was recorded at $0,1,4$ and 6 min. The scavenging capacity of test compounds was calculated using the following equation: $\mathrm{ABTS}^{\circ+}$ scavenging effect $(\%)=100\left(\mathrm{~A}_{0}-\right.$ $\left.A_{c}\right) / A_{0}$, where $A_{0}$ is absorbance of a control lacking any radical scavenger and $A_{c}$ is absorbance of the remaining $\mathrm{ABTS}^{*+}$ in the presence of scavenger. Graphs of antioxidant concentration versus percent absorbance reduction were then plotted. The concentration of Trolox giving the same percentage reduction of absorbance at $734 \mathrm{~nm}$ as the $1 \mathrm{mM}$ antioxidant solution was calculated from the three point graphs. The results were thus expressed as Trolox equivalent antioxidant capacity (TEAC) values. For each hydrazone and each concentration, measurements were performed in triplicate. Absorbance values were corrected for radical decay using blank solutions.

\subsection{Biochemical experiments}

\section{LDL oxidation}

LDL were isolated by ultracentrifugation from the pooled plasma of healthy normolipidemic human subjects and dialyzed against PBS containing $100 \mu \mathrm{mol} / \mathrm{L}$ of ethylenediaminetetraacetic acid (EDTA), as previously indicated [11]. LDL oxidation and the 
antioxidant effect of syringic hydrazones were evaluated using three different protocols, two cell-free systems (copper- and UV-mediated oxidation) and one cell-mediated oxidation system by HMEC-1. Trolox $(10 \mu \mathrm{M})$ was used as reference inhibitor of LDL oxidation, for the 3 methods. The extent of LDL oxidation was monitored by the determination of the thiobarbituric acid reactive substances (TBARS) content in the culture medium after $6 \mathrm{~h}$ incubation. The amount of TBARS formed in the absence of any agent was: cell-mediated oxidation, 10.2-15.4 nmol TBARS/mg apoB; UV-induced oxidation, 6.4-9.7 nmol TBARS/mg apoB; copper-mediated LDL oxidation, 21.6-27.8 nmol TBARS/mg apoB. The results are expressed as $\%$ of TBARS formed in the presence of the different agents vs TBARS formed in the absence of antioxidants.

- Copper-induced LDL oxidation: LDL solutions (0.5 mg apoB/mL $\mathrm{NaCl} 150 \mathrm{mM}, \mathrm{pH} 7.3$ ) were incubated for 3 hours at $37^{\circ} \mathrm{C}$, in the presence of $\mathrm{CuSO}_{4} 5 \mu \mathrm{M}$, as reported [11-23] and of the different syringic hydrazones used at the fixed concentration of $10 \mu \mathrm{M}$. At the end, the oxidation of LDL was monitored by the determination of the TBARS content, using the fluorimetric procedure of Yagi [24].

- UV-induced LDL oxidation: LDL solutions (1 mg apoB/mL NaCl $150 \mathrm{mM}$, pH 7.3 containing or not syringic hydrazones, $10 \mu \mathrm{M}$ ) were irradiated for 2 hours, as a thin film (5 $\mathrm{mm}$ ) in an open beaker placed $10 \mathrm{~cm}$ under the UV-C source (HNS 30W OFR Osram UV-C tube, $\lambda \square \max 254 \mathrm{~nm}, 0.5 \mathrm{~mW} / \mathrm{cm}^{2}$ ) [11]. At the end, the TBARS content was determined.[24] - Cell-mediated LDL oxidation: HMEC-1 diluted in DMEM (Dulbecco's modified Eagle's medium) cultured medium containing $10 \%$ foetal calf serum and antibiotics were seeded in 24 multiwell plates. After $24 \mathrm{~h}$, the standard culture medium (on sparse proliferative cells), was removed and replaced by serum-free Roswell Park Memorial Institute (RPMI-1640) medium containing native LDL $\left(100 \mu \mathrm{g}\right.$ apoB/mL) and $\mathrm{CuSO}_{4}(1 \mu \mathrm{M})$, and HMEC-1. This mixture 
was incubated at $37^{\circ} \mathrm{C}$ for 6 hours, in the presence (or the absence) of syringic hydrazones or Trolox (10 $\mu \mathrm{M}$ each) [11-24]. The same experiment was also conducted for syringaldehyde, hydralazine, isoniazid and aminoguanidine. At the end of the incubation, the LDL-containing medium was used for determining TBARS [24]. The results were expressed as $\% \pm$ S.E. of TBARS content in LDL oxidized in the absence of antioxidant. Statistical analyses were performed by ANOVA (Tukey test - SigmaStat software).

\section{Cytoprotective and carbonyl scavenger effect}

The subsequent toxicity of cell-oxidized LDL (in the absence or presence of the different agents), was evaluated on HMEC-1 after 24h incubation, using the MTT test $[11,24]$. Results were expressed as \% of cell viability vs the unstimulated control (without LDL). The residual cell viability in cell incubated with LDL in the absence of agents was around $15-21 \%$. The carbonyl scavenger effect of the various compounds was undirectly measured by evaluating their cytoprotective effect on the cytotoxicity induced by 4-hydroxynonenal (4-HNE), an aldehyde formed during the peroxidation of polyunsaturated fatty acids, and involved in the formation of adducts on proteins. The effect of the newly synthesized agents was compared to that observed with Trolox, a classical antioxidant unable to block 4-HNE-induced cytotoxicity.

\section{Superoxide anion generation}

Superoxide anion $\left(\mathrm{O}_{2}{ }^{--}\right)$production was measured as the superoxide dismutase (SOD)-inhibit able reduction of cytochrome $\mathrm{C}$ and expressed as $\%$ of $\mathrm{O}_{2}{ }^{--}$generated in HMEC-1 incubated 1h with LDL in the presence of agents vs HMEC-1 incubated with LDL alone (8.2-10.6 $\mathrm{nmol} / \mathrm{h} / \mathrm{mg}$ protein). All these data are a mean of 6 separate experiments +/-SEM. Briefly, sub confluent HMEC-1 were pre-incubated for 30 and 60 min, in $1 \mathrm{~mL}$ phenol red free RPMI 
containing cytochrome $\mathrm{C}(20 \mu \mathrm{M})$ with or without SOD $(100 \mathrm{mU} / \mathrm{L}), \mathrm{LDL}(100 \mu \mathrm{g}$ apoB/mL) and the different hydrazones or Trolox $(10 \mu \mathrm{M})$. The $\mathrm{O}_{2}{ }^{\circ-}$ specific reduction of cytochrome $\mathrm{C}$ was determined spectrophotometrically at $550 \mathrm{~nm}$ (difference in absorbance with or without SOD), according to McCord and Fridovitch [24] and under the previously used conditions $[23]$.

4-HNE-induced cytotoxicity

Subconfluent HMEC-1 were overnight incubated in serum-free Hank's medium in the presence of 4-HNE $(25 \mu \mathrm{M})$, and the different molecules tested at a fix concentration of 10 $\mu \mathrm{M}$. The cytotoxic effect of 4 -HNE was evaluated by MTT. Data are expressed as $\%$ of the untreated control. Statistical analysis was performed by ANOVA (Tukey test - SigmaStat software).

\section{Acknowledgement}

The authors acknowledge the financial support from INSERM, CNRS, Université Paul Sabatier and COST B35. Nadji Belkheiri is recipient of a grant from algerian government. Benaissa Bouguerne is recipient of the Ministère de l'Enseignement Supérieur et de la Recherche.

\section{References}

[1] H. Sies, Am. J. Med. 91 (1991) 31S-38S.

[2] H. Esterbauer, R.J. Schaur, H. Zollner, Free Radic. Biol. Med. 11 (1991) 81-128.

[3] K. Uchida, Free Radic. Biol. Med. 28 (2000) 1685-1696.

[4] G. Leonarduzzi, M.C. Arkan, H. Basaga, E. Chiarpotto, A. Sevanian, G. Poli, Lipid Free Radic. Biol. Med. 28 (2000)1370-1378.

[5] E.R. Stadtman, Ann. N Y Acad. Sci. 928 (2001) 22-38. 
[6] A. Negre-Salvayre, C. Coatrieux, C. Ingueneau, R. Salvayre, Br. J. Pharmacol. 153 (2008) 6-20.

[7] D. Steinberg, J. Biol. Chem. 272 (1997) 20963-20966.

[8] A.J. Lusis, Nature 407 (2000) 233-241.

[9] R. Stocker, Curr. Opin. Lipidol. 10 (1999) 589-597.

[10] R. Brigelius-Flohe, D. Kluth, A. Banning, Mol. Nutr. Food Res. 49 (2005) 1083-1089.

[11] S. Galvani, C. Coatrieux, M. Elbaz, M.H. Grazide, J.C. Thiers, A. Parini, K. Uchida, N. Kamar, L. Rostaing, M. Baltas, R. Salvayre, A. Nègre-Salvayre, Free Radic. Biol. Med. 45 (2008) 1457-1467.

[12] C. Lapeyre, M. Deloménède, F.Bedos-Belval, H. Duran, A. Negre-Salvayre; M. Baltas, J. Med. Chem. 48 (2005) 8115-8124.

[13] M. Delomenède, F. Bedos-Belval, H. Duran, C. Vindis, M. Baltas, A. Nègre-Salvayre, J. Med. Chem. 51 (2008) 3171-3181.

[14] M.C. Foti, C. Daquino, C. Geraci, J. Org. Chem. 69 (2004) 2309-2314.

[15] N. Nenadis, L-F. Wang, M. Tsimidou, H-Y. Zhang, J. Agric. Food Chem. 52 (2004) 4669-4674.

[16] R. Re, N. Pellegrini, A. Proteggente, A. Pannala, M. Yang, C. Rice-Evans, Free Radic. Biol. Med. 26 (1999) 1231-1237.

[17] M. Harej, D. Dolenc, J. Org. Chem. 72 (2007) 7214-7221.

[18] J.W. Heinecke, M. Kawamura, L. Suzuki, A. Chait, J. Lipid Res. 34 (1993) 20512061.

[19] O. Ziouzenkova, A. Sevanian, P.M. Abuja, P. Ramos, H. Esterbauer, Free Radic. Biol. Med. 24 (1998) 607-623.

[20] I. Pinchuk, D. Lichtenberg, Free Radic. Res. 24 (1996) 351-360.

[21] R. Albertini, P.M. Abuja, Free Radic. Res. 30 (1999) 181-178. 
[22] P. Molyneux, Songklanakarin J. Sci. Technol. 26 (2004) 211-219.

[23] L. Mabile, O. Meilhac, I. Escargueil-Blanc, M. Troly, M.T. Pieraggi, R. Salvayre, A. Nègre-Salvayre, Arterioscler. Thromb. Vasc. Biol. 17 (1997) 1575-1582.

[24] K. Yagi, Chem. Phys. Lipids. 45 (1987) 337-351.

[25] J.M. McCord, I. Fridovich, J. Biol. Chem. 244 (1969) 6049-6055.

[26] S. De, S. Adhikari, J. Tilak-Jain, V.P. Menon, T.P. Devasagayam, Chem. Biol. Interact. 17 (2008) 215-223. 
Figures, Schemes and Tables

Scheme 1. Synthesis of syringic hydrazones.

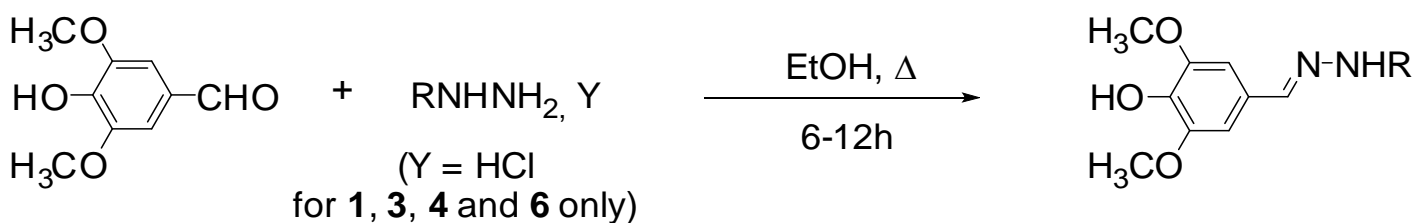

\section{Cpd}

ID

12

34

5

$\begin{array}{lll}6 & 7 & 8\end{array}$

9

$\mathbf{R}$<smiles>Cc1nncc2ccccc12</smiles><smiles>Cc1nc2ccccc2s1</smiles><smiles>Cc1ccccc1F</smiles><smiles>CCc1ccc(C)cc1</smiles><smiles>Cc1cc(Cl)c(Cl)cc1F</smiles><smiles>NC1CCCC1</smiles><smiles>CC(=O)c1cccnc1</smiles><smiles>CC(=O)c1ccncc1</smiles><smiles>CC(=O)c1cc(C)n[nH]1</smiles><smiles>CC(=O)Oc1ccccc1</smiles>

Yield $\quad 75 \%$

$82 \%$ $47 \%$

$48 \%$ $86 \%$ $89 \% \quad 97 \%$ $64 \%$ $90 \%$

Figure 1. IC50 : concentration needed for reducing $\mathrm{DPPH}^{\circ}$ absorption by $50 \%$ at $517 \mathrm{~nm}$.

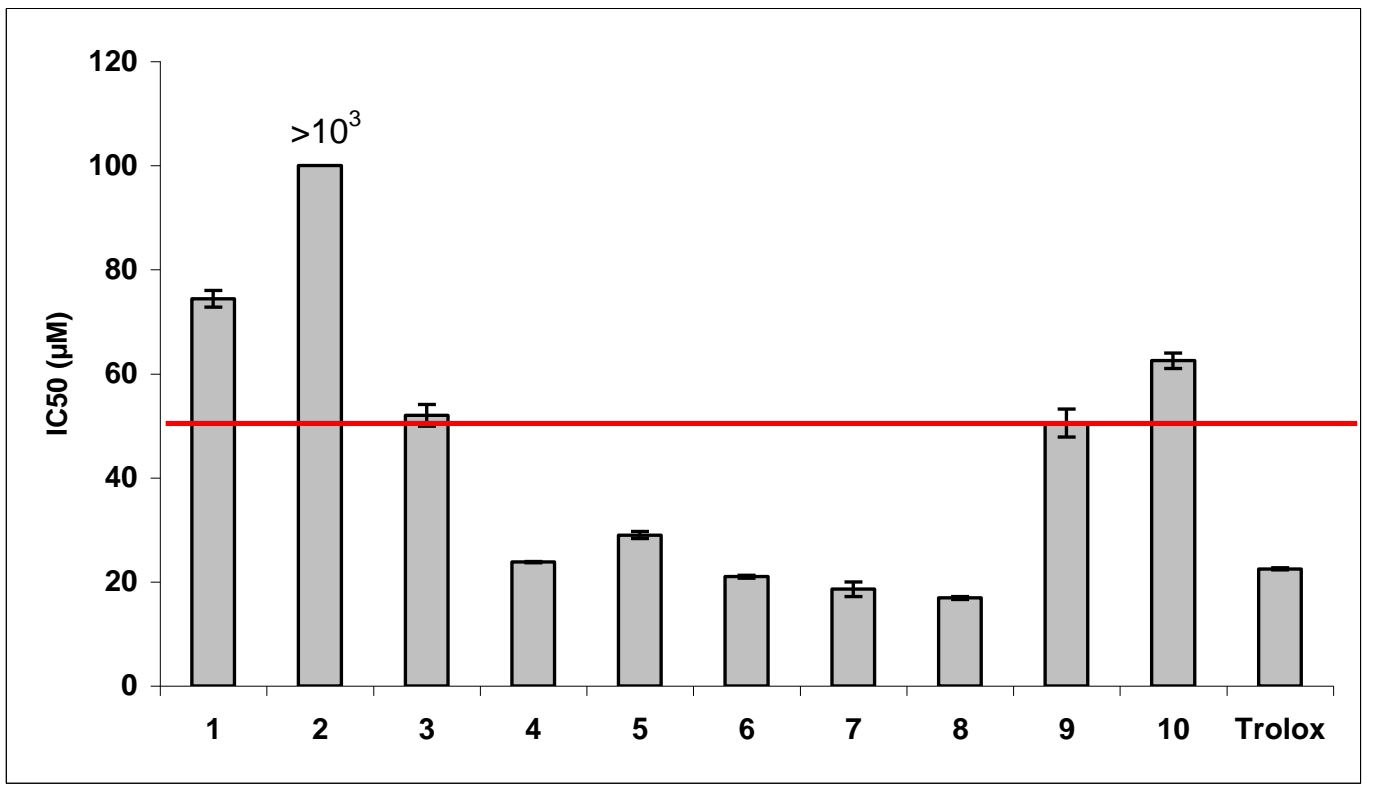

Values are average of 3 independent measurements for each compound. Errors are $\pm \log S D$ (standard deviation). 
Scheme 2. Purposed reaction of $\mathrm{DPPH}^{\bullet}$ with nicotinohydrazide derivative $\mathbf{8}$.

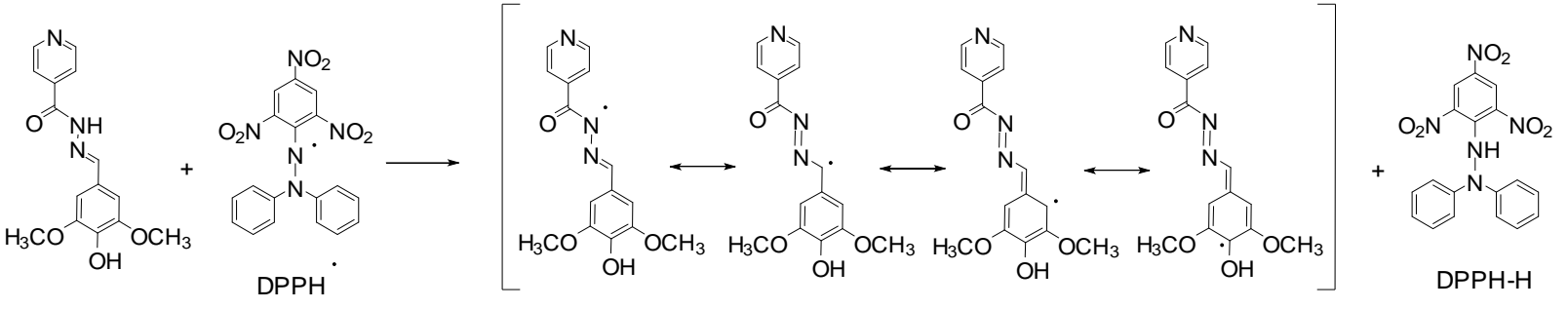

(E)-N'-(4-hydroxy-3,5-dimethoxybenzylidene)nicotinohydrazide intermediate radicals

Figure 2. ABTS Radical scavenging activity of syringic hydrazones compared with Trolox.

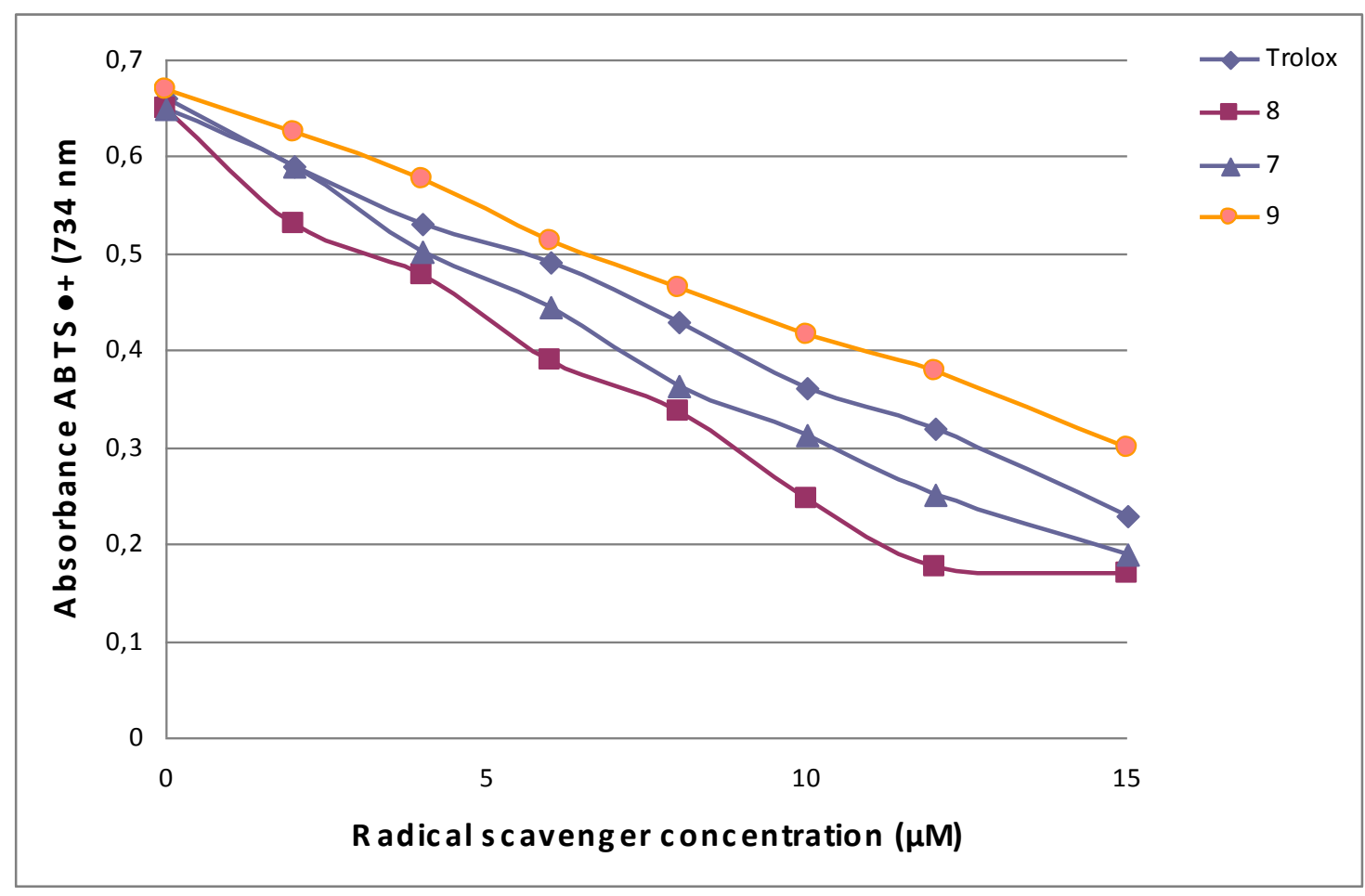

Average values of 3 independent measurements $\pm \log S D$ for each compound. 
Table 1. Determination of antioxidant activity as TEAC of syringic hydrazones by ABTS.

\begin{tabular}{cc}
\hline Cpd ID & TEAC \\
\hline $\mathbf{1}$ & $0.75 \pm 0.1$ \\
$\mathbf{2}$ & $0.89 \pm 0.01$ \\
$\mathbf{3}$ & $0.78 \pm 0.016$ \\
$\mathbf{4}$ & $1.02 \pm 0.01$ \\
$\mathbf{5}$ & $0.98 \pm 0.03$ \\
$\mathbf{6}$ & $0.69 \pm 0.02$ \\
$\mathbf{7}$ & $1.25 \pm 0.024$ \\
$\mathbf{8}$ & $1.25 \pm 0.02$ \\
$\mathbf{9}$ & $1.15 \pm 0.02$ \\
$\mathbf{1 0}$ & $1.11 \pm 0.024$ \\
Trolox & 1 \\
\hline
\end{tabular}

Average values $\pm \log S D$ of 3 independent measurements for each compound. 
Table 2. Determination of protective activity of syringic hydrazones on LDL oxidation.

\begin{tabular}{cccccc}
\hline Cpd ID & $\begin{array}{c}\text { LDL oxidation } \\
\text { In vitro: Cu }\end{array}$ & $\begin{array}{c}\text { LDL oxidation } \\
\text { In vitro: } \mathbf{U V}\end{array}$ & $\begin{array}{c}\text { LDL oxidation } \\
\text { Cell-mediated }\end{array}$ & MTT & O $^{{ }^{*}}$ \\
\hline $\mathbf{1}$ & $5 \pm 1.5$ & $18 \pm 2.5$ & $4 \pm 0.2$ & $66 \pm 5$ & $30 \pm 3$ \\
$\mathbf{2}$ & $2 \pm 0.3$ & $17 \pm 0.3$ & $3 \pm 0.2$ & $55 \pm 1$ & $56 \pm 4$ \\
$\mathbf{4}$ & $4 \pm 0.1$ & $16 \pm 3.5$ & $13 \pm 9$ & $79 \pm 1$ & $33 \pm 2$ \\
$\mathbf{5}$ & $6 \pm 0.3$ & $18 \pm 2.5$ & $5 \pm 0.3$ & $58 \pm 6$ & $57 \pm 6$ \\
$\mathbf{6}$ & $3 \pm 0.1$ & $15 \pm 3.5$ & $4 \pm 0.3$ & $74 \pm 4$ & $42 \pm 3$ \\
$\mathbf{7}$ & $6 \pm 0.2$ & $24 \pm 1.5$ & $10 \pm 6$ & $70 \pm 1$ & $36 \pm 3$ \\
$\mathbf{8}$ & $77 \pm 6$ & $20 \pm 1.5$ & $5 \pm 0.1$ & $60 \pm 10$ & $44 \pm 3$ \\
$\mathbf{9}$ & $45 \pm 7$ & $18 \pm 1.5$ & $7 \pm 0.2$ & $65 \pm 7$ & $22 \pm 2$ \\
$\mathbf{1 0}$ & $6 \pm 0.3$ & $17 \pm 1.5$ & $3 \pm 0.1$ & $69 \pm 0.2$ & $50 \pm 3$ \\
\hline Trolox & $6 \pm 0.2$ & $18 \pm 3.5$ & $3 \pm 0.2$ & $69 \pm 9$ & $50 \pm 4$ \\
\hline & & $18 \pm 4.5$ & $3 \pm 0.1$ & $57 \pm 0.1$ & $5 \pm 2$ \\
\hline
\end{tabular}


Figure 3. Cell-mediated LDL oxidation and subsequent toxicity on HMEC-1 cells of syringic hydralazine 1, syringic aminoguanidine 6 and syringic isoniazid 8. Comparison with their constitutive frames.

A

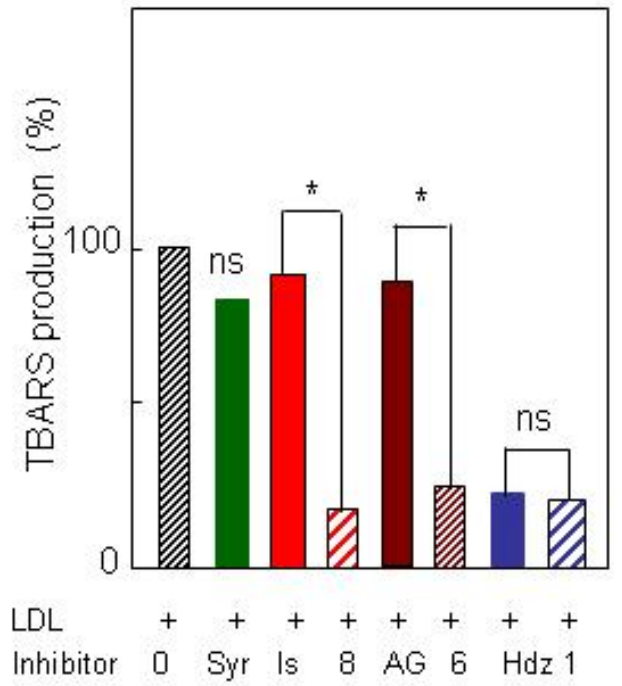

B

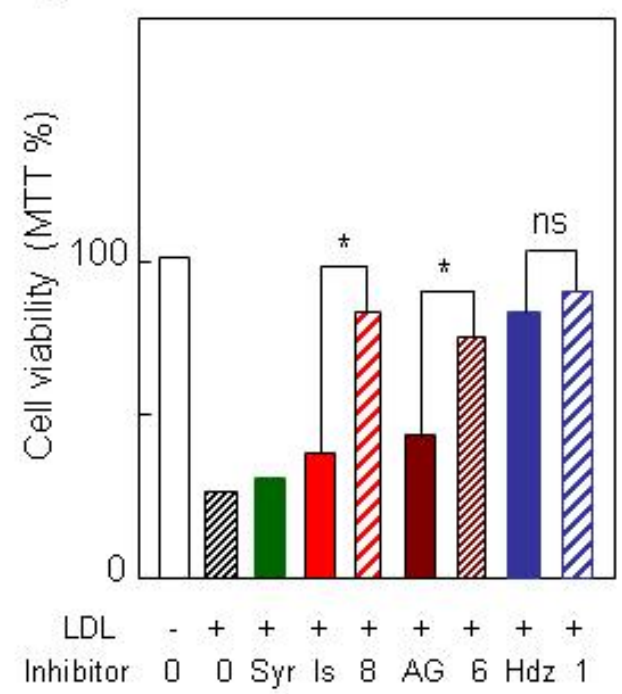

* significant between the indicated values. $n s$ : not significant between the indicated values.

Syr, syringaldehyde; Is, isoniazid; AG, aminoguanidine; Hdz, hydralazine. 
Figure 4. Cytoprotective effect of syringic hydrazones against 4-HNE-induced cytotoxicity.

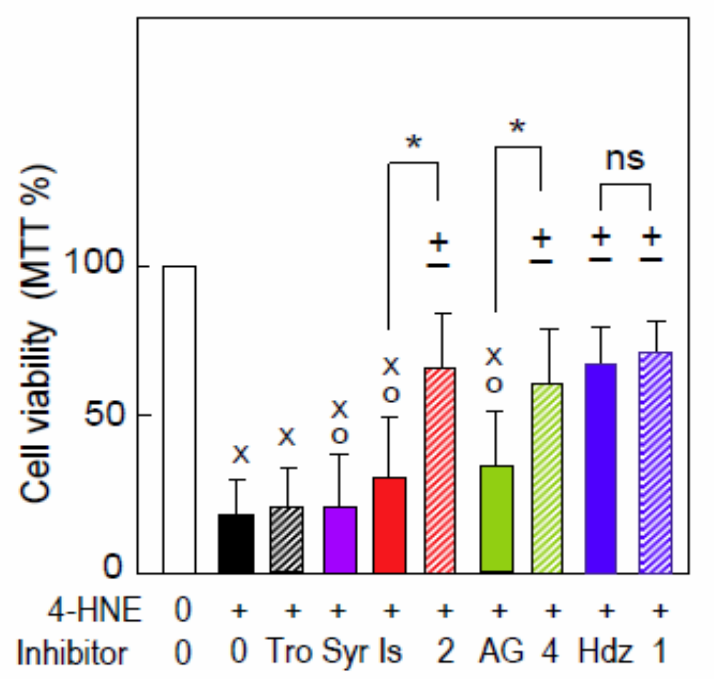

* Significant between the indicated values. $n s$ : not significant between the indicated values.

Syr, syringaldehyde; Is, isoniazid; AG, aminoguanidine; Hdz, hydralazine; Tro, Trolox. 